\title{
Modeling the Dynamics of Driver's Dilemma Zone Perception Using Agent Based Modeling Techniques
}

\author{
Montasir Abbas, Ph.D., P.E. ${ }^{1}$ and Sahar Ghanipoor Machiani, Ph.D. ${ }^{2}$ \\ ${ }^{1}$ Associate Professor, Department of \\ Civil and Environmental Engineering, Virginia Tech \\ ${ }^{2}$ Research Associate, Virginia Tech \\ Transportation Institute \\ 1abbas@vt.edu, 2sahargh@vt.edu
}

\begin{abstract}
Several research efforts investigated and targeted the issue of reducing dilemma zone (DZ) related crashes (where drivers upstream of intersections are uncertain about their decision to stop or go at the onset of yellow). One of the important unanswered questions in the literature is whether driver's perception of DZ changes individually as a function of their safe and unsafe past experience at similar situations. This paper investigates the use of agent-based methods in capturing the effect of driver's learning/dynamic perception of DZ. A driving simulator was used to collect driver behavior data. An actor-critic reinforcement learning algorithm was implemented to model the dynamic behavior of driver in dilemma zone. Fuzzy logic is used to partition traffic state variables and a reinforcement learning technique is used in policy calibration and update. The study results show a close matching between the driver's action from the driving simulator and the model output.

The research reported here contributes to improved modeling of driver definition and behavior in dilemma zone, which will have significant impacts on the design of optimal control methods and the assessment of intersection safety. Moreover, it lays the groundwork for several subsequent simulator studies and scenario development in driving simulator to investigate the drivers' behavior at signalized intersections.
\end{abstract}

Keywords: Dilemma zone; machine learning; dynamic perception; driving simulator

\section{Introduction}

Around 45 percent of all crashes in the United States take place at intersections, of which, a majority is related to drivers decisions at Dilemma Zone (DZ) [1-3]. Generally, dilemma zone is defined as the area where drivers approaching the intersection must decide to either proceed or stop at the onset of the yellow indication [4]. Drivers that might perceive themselves to be too close to an intersection for a safe stop, and too far to proceed without violating traffic regulations, are said to be caught in DZ [5]. Avoiding improper decisions to brake hard in response to a yellow signal indication (leading to rear-end crashes) or to proceed into the intersection without being able to clear it before the beginning of red (leading to red-light running incidents and possibly right-angle crashes) can be achieved by designing the signal operation such that the number of drivers caught in DZ is minimized. There are other influencing factors such as traffic composition (i.e., cars and trucks), pavement condition, and the grade of the roadway, which make it especially important to address DZ safety issues with better modeling of driver behavior.

One of the important unanswered questions in the literature is whether driver's perception of DZ changes individually as a function of their past experience at signalized intersection (their perception of whether their behavior at an intersection was safe or unsafe). In other words, whether driving through safer intersections could be decreasing 
the alertness of drivers to possible DZ issues, and thus exposes them to more severe crashes at other intersections. Therefore, it would be useful to model and quantify the changes in drivers' DZ perception, and corresponding actions, as a function of their past experience in DZ.

This paper investigates the use of machine learning/agent-based methods in capturing the effect of driver's learning/dynamic perception of DZ. Data for the analysis is obtained from a driving simulator study. The data is used to investigate the potential of using a reinforcement learning model to model the driver decision in DZ. The results of this study contribute to the improvement of dilemma zone modeling and designing potential protection and mitigation strategies.

The paper begins with a review of relevant research. In the following sections the experimental design of the driving simulator experiment is explained including the description of the collected data. A description of machine learning model and results then follows. The final section offers concluding remarks and future directions.

\subsection{Review of Relevant Literature}

Dilemma zone and drivers' decision has been the subject of research for decades. Two types of dilemma zones are recognized in the literature [6-11]. Type I is defined as an area where the driver can neither stop without slamming on the brakes or clear the intersection safely without running the red light. Type II (option zone) is associated with drivers' different decisions and defined as the area where the driver can not easily determine whether stop or cross successfully based on his/her perception.

Various factors are recognized in the literature that affects driver behavior, and the decision making process at the onset of the yellow indication. These factors are categorized in 5 main categories, namely: (1) Driver's attributes, (2) Intersection characteristics and condition, (3) Subject vehicle characteristics, (4) Signal control settings, and (5) Traffic flow characteristics. These influencing factors play an important role in driver behavior modeling which is discussed here.

Probability of stopping in the earlier studies was usually determined based upon limited number of factors such as distance to intersection [12-16], time to intersection [17, 18], and approaching speed [17, 19]. Other factors started to be taken into account to better estimate the drivers' behavior at the onset of yellow indication. Research efforts in this regard fall into three main groups of probabilistic, statistical, and fuzzy models as described below.

$\mathrm{Li}$ and Abbas [20] introduced a dilemma hazard model based upon the previous studies on drivers' response to the yellow onset, vehicle kinematics, and a Monte Carlo simulation framework. The proposed model assigns a hazard weight to each vehicle located in dilemma zone. The model was calibrated and validated with the vehicle trajectory data collected at a high-speed signalized intersection.

Olson and Rothery [12] determined the probability of stopping as a function of distance to the intersection for five intersections with different speed. Chang, Messer, \& Santiago[13] conducted a study based on collected data on seven sites and suggested a new perception reaction time and use of constant yellow interval of 4.5 seconds.

Allos \& Al-Hadithi [4] developed a model of drivers' behavior by capturing the relationship between the likelihood of stopping and going and the influential factors including the ratio of secondary traffic flow to the main traffic, yellow duration, speed, distance, number of intersection arms, and Surveillance police. They found that police presence does not have a direct influence on the likelihood of stopping. Although, it influences the dependent variable indirectly as significant correlation is recognized between police presence and approaching speed [4].

Gates, Noyce, Laracuente, and Nordheim [21] modeled deceleration rate and brakeresponse time statistically. The estimated travel time to the intersection at the onset of yellow indication turned out to be the most important influential factor on a driver's 
likelihood to stop or go. Caird, Chisholm, Edwards, and Creaser [22] estimated drivers stopping or running decision based on time to stop line. The main focus of their study was to evaluate the effect of age group on perception reaction time. They observed no age differences in perception reaction time.

Elmitiny, Yan, Radwon, Russo, and Nashar [7] conducted a statistical analysis, specifically Tree-based classification analysis, on video-based system collected data and concluded that the most important predictors for both the stop/go decision and red-light running violation are vehicle's distance from the intersection, operating speed, and position in the traffic flow. Sharma, Bullock, and Peeta [23] tested five variables including required acceleration and deceleration rates. According to this study, acceleration required by the vehicle to cross the stop bar prior to onset of red turned out to be a significant factor in drivers' decision of stopping or going.

Applying the field data Kikuchi, Perincherry, Chakroborty, and Takahashi [24] modeled the drivers' decision considering a set of fuzzy inference rules for stopping or passing through the intersection. They estimated the degree of anxiety for aggressive and conservative drivers using Yager's anxiety measure [25]. Anxiety level is the degree of uncertainty the driver experiences in making a correct decision to stop or go [26]. Based on a rule-based fuzzy logic system Lin \& Kuo [26] introduced a procedure to estimate the change and clearance intervals of a traffic signal. The last example in this part is a study by Hurwitz, Wang, Knodler Jr., Ni, and Moore [11]. They developed a binary logistic regression model for drivers' probability of stopping or going. The input for the model is generated from a fuzzy subset that requires less data compared to similar models.

Despite the vast body of related literature, a shortcoming observed in the body of the literature is paying no attention to the "dynamic nature of drivers' decision". Looking at the dynamic behavior means how the dilemma zone definition changes as a function of drivers' safe and unsafe experience. In other words, one important question that remains unanswered in literature is whether the DZ definition changes individually as a function of experience. A major concern is whether driving through safer intersection could be reducing the alertness of drivers to possible DZ issues, and therefore setting them up for more severe crashes at other intersections. Alternatively, one would like to be able to model and quantify the changes in DZ definition in individual drivers as a function of their safe and unsafe experience, including the ability to quantify the benefits of training/educating drivers about DZ issues. It is of vital importance to investigate the effect of drivers' experience as it contributes to determining the benefits of training drivers about dilemma zone issues. This study fills a gap in the literature about drivers' learning aspect of dilemma zone by designing an adaptive experimental plan for a driving simulator study and investigating the use of machine learning methods in capturing the effect of driver's dynamic perception of DZ.

\section{Experimental Design of Driving Simulator Experiment}

A driving simulator study was designed to test the significance of several factors, identified in the literature as important factors, as well as the learning aspects of drivers. Thirty four subject drivers participated in the study. The demographic characteristics of participants are shown in Table 1. 
Table 1. Demographic Characteristics of Participants

\begin{tabular}{lllllllllll}
\hline Age Group (years) & $18-22$ & $23-24$ & $25-27$ & $28-33$ & \multicolumn{2}{c}{$34-68$} \\
\cline { 2 - 12 } & M & F & M & F & M & F & M & F & M & F \\
\hline VA & - & 2 & 4 & - & 1 & 1 & 2 & 2 & 3 & 1 \\
\hline MD & - & 1 & 1 & 1 & 2 & - & - & - & - & - \\
\hline PA & 1 & - & - & - & - & - & - & - & - & 2 \\
\hline Other & 2 & 1 & - & 1 & 2 & - & 3 & - & 1 & - \\
\hline
\end{tabular}

Experimental factors included in the study were:

1. Time to intersection,

2. Presence of police,

3. Whether the pavement is wet or dry,

4. Presence of a traffic around the subject car, and

5. Presence of queues at the side street of the intersection

\subsection{Factor Levels}

The intersection factor and their levels used in the experiment were as follows:

- Time to intersection (TTI) at the onset of yellow (s)

Levels: $2.5,3.5,4.5$

- $\quad$ Presence of police

Levels: Yes, No

- $\quad$ Pavement condition

Levels: Wet, Dry

- Other vehicle around

Levels: No Vehicle, Back

- $\quad$ Presence of side street queue

Levels: Yes, No

\subsection{Experiment Adaptation Factor}

Since the primary goal of this study is to investigate the drivers' learning process while driving through safe and unsafe intersections, two driving learning factors were included in the experiment. These factors were specifically designed to test whether drivers changed their behavior after being exposed to a long yellow duration and after running a red light, and driving through an intersection that implements a DZ protection algorithm (by incrementing each factor after the driver is exposed to one of those situations). The experiment adaptation situations, hypotheses tested, and rationale behind the hypotheses are summarized in Table 2. A complete description of the experimental design including all factors could be found in [27]. 
Table 2. Experiment Adaption Situations and Rationale

\begin{tabular}{|c|c|c|c|}
\hline Situation & $\begin{array}{l}\text { Experiment } \\
\text { Adaptation } \\
\end{array}$ & Rationale & Hypothesis Tested \\
\hline \multicolumn{4}{|l|}{$\begin{array}{l}\text { Yellow is presented at } \\
\text { different driver's } \\
\text { TTIs }\end{array}$} \\
\hline $\begin{array}{l}\text { a. Driver decides to } \\
\text { stop. }\end{array}$ & $\begin{array}{l}\text { Increase yellow } \\
\text { duration while the } \\
\text { driver is waiting at } \\
\text { the stop bar. }\end{array}$ & $\begin{array}{l}\text { Investigate } \\
\text { whether a driver } \\
\text { would tend to go } \\
\text { in a similar } \\
\text { situation due to } \\
\text { their realization } \\
\text { that they could } \\
\text { have passed } \\
\text { safely with such a } \\
\text { long yellow. }\end{array}$ & $\begin{array}{l}\text { Driver behaviordoes } \\
\text { not change. }\end{array}$ \\
\hline $\begin{array}{l}\text { b. Driver decides to } \\
\text { pass. }\end{array}$ & $\begin{array}{l}\text { Decrease yellow } \\
\text { duration so that the } \\
\text { driver ends up } \\
\text { running the red } \\
\text { light. }\end{array}$ & $\begin{array}{l}\text { Investigate } \\
\text { whether the } \\
\text { driver would tend } \\
\text { to stop in a } \\
\text { similar situation } \\
\text { due to their } \\
\text { realization } \\
\text { thatthey might } \\
\text { end up running } \\
\text { the red light with } \\
\text { their existing } \\
\text { perception of DZ. }\end{array}$ & $\begin{array}{l}\text { Driver behaviordoes } \\
\text { not change. }\end{array}$ \\
\hline
\end{tabular}

Two levels of "Do nothing" and "Do something are considered for the "experiment adaptation factor". "Do nothing" level relates to the condition that no "experiment adaptation factor" is examined at an intersection; meaning that a normal yellow duration is implemented. "Do something" refers to the case that one of the experiment adaptation approach defined in table 1 is considered based upon drivers' behavior. The flowchart of the "experiment adaptation factor" is illustrated in Figure 1.

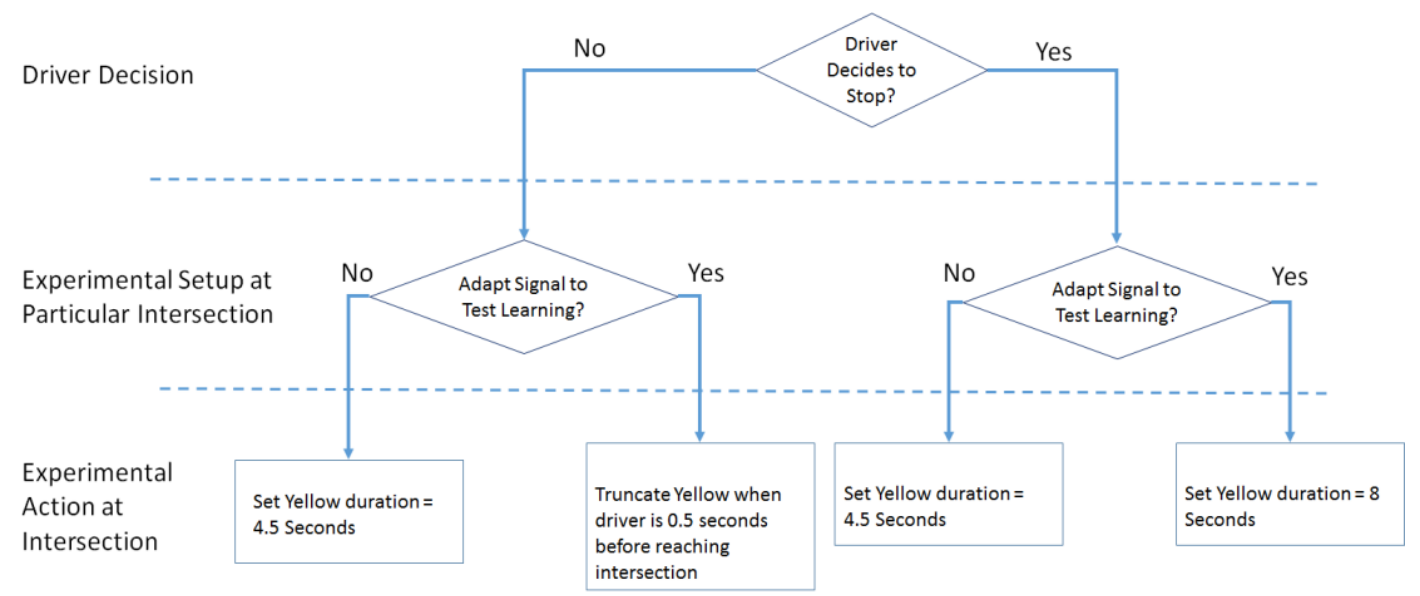

Figure 1. Flowchart of the Experiment Adaptation 


\section{Scenario Implementation in the Driving Simulator}

A DriveSafety DS-250 model driving simulator (fixed-based with no motion cues) was used in this study. The simulator contains three major components, namely Vection ${ }^{\mathrm{TM}}$ (high fidelity real-time driving simulation software), HyperDrive (advanced scene and scenario authoring tool set), and Dashboard (Software that interfaces Vection and Hyperdrive). The driving simulator and two screenshots of red and yellow signal indication in the simulator environment are shown in Figure 2.
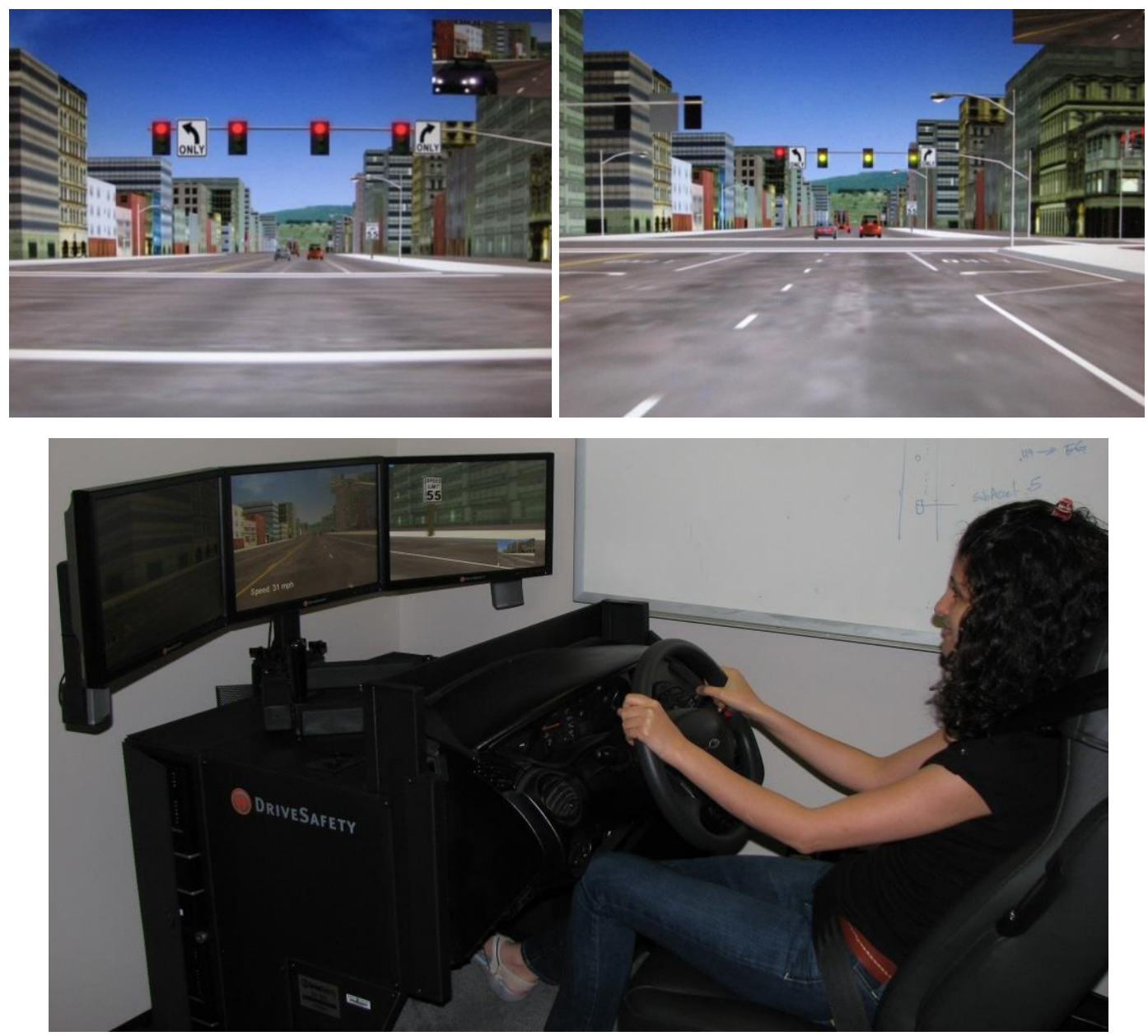

Figure 2. Driving Simulator

Scenario design and development was handled in Hyperdrive. Basic elements of the driving environment such as road structure, scenery, signage, and other static items are implemented using drag and drop assembly of database elements from the built-in library. However, more complicated settings such as traffic signal alteration and accident setups need to be coded in TCL (Tool Command Language) format. Driving simulator data is obtained from two sources of built-in data (i.e., variables are selected from a predefined list) and user-defined data (i.e., variables are defined by user in the script).

Based upon our experimental design, participants drive through 5 different scenarios, each including 10 intersections. There are specific events occurring at certain time or location to intersections that need to be implemented in the simulator. Time triggers (triggers that gets activated when the subject car is at a particular time) and location triggers (triggers that gets activated when the subject car is at a particular location) were created and programmed appropriately to account for these events. In total, three location trigger and one time trigger were created at the start of each scenario, and six time triggers 
and three location triggers were implemented at each intersection. Triggers at each intersection are shown in Figure 3. Figure 3-a shows how the layout looks like in HyperDrive environment, and Figure 3-b provides a plan of triggers' sequence (time triggers and location triggers are coloured differently; dark purple shows location triggers and light purple is associated with time triggers). Applications of these triggers are summarized in Table 2.

Table 2. Trigger Applications

\begin{tabular}{|c|l|l|l|}
\hline $\begin{array}{c}\text { Trigger } \\
\text { number }\end{array}$ & Trigger name & $\begin{array}{l}\text { Trigger } \\
\text { type }\end{array}$ & Application \\
\hline 1 & $\begin{array}{l}\text { DoSomething time } \\
\text { trigger }\end{array}$ & Time & $\begin{array}{l}\text { Located 6 sec ahead of the intersection to } \\
\text { implement the “experiment adaptation factor" }\end{array}$ \\
\hline 2 & TouchBrakeStart & $\begin{array}{l}\text { Located 5.5 sec ahead of the intersection to } \\
\text { start monitoring the attempt of drivers to } \\
\text { initiate a stop }\end{array}$ \\
\hline 3 & TouchBrakeEnd & $\begin{array}{l}\text { Tocated 4 sec ahead of the intersection to } \\
\text { terminate monitoring the attempt of drivers to } \\
\text { initiate a stop }\end{array}$ \\
\hline 5 & YellowSignal & $\begin{array}{l}\text { Located 4.5, 3.5, or 2.5 sec ahead of the } \\
\text { intersection to change the signal to yellow } \\
\text { based on the selected time to intersection level }\end{array}$ \\
\hline 6 & RedLightRunner & Time & $\begin{array}{l}\text { Located 1.5 sec ahead of the intersection to } \\
\text { change the signal to yellow }\end{array}$ \\
\hline 7 & StopGoDecision & $\begin{array}{l}\text { Located 0.5 sec ahead of the intersection to } \\
\text { change the signal to red (to implement red- } \\
\text { light running scenarios when appropriate) }\end{array}$ \\
\hline 9 & CameraFlash & $\begin{array}{l}\text { Located 17 meters ahead of the intersection to } \\
\text { recognize the driver's decision at the } \\
\text { intersection }\end{array}$ \\
\hline
\end{tabular}

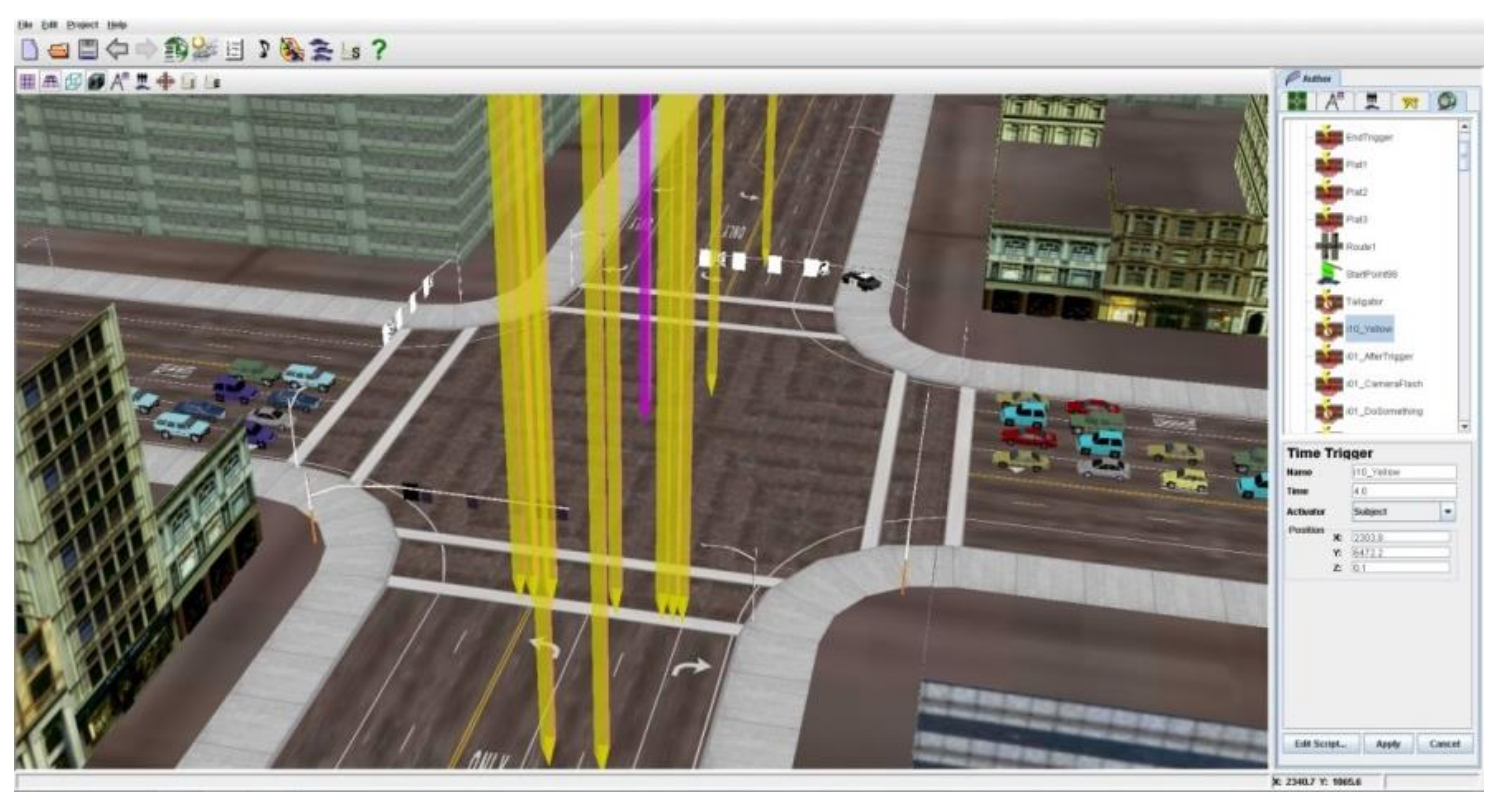

a) HyperDrive Layout 


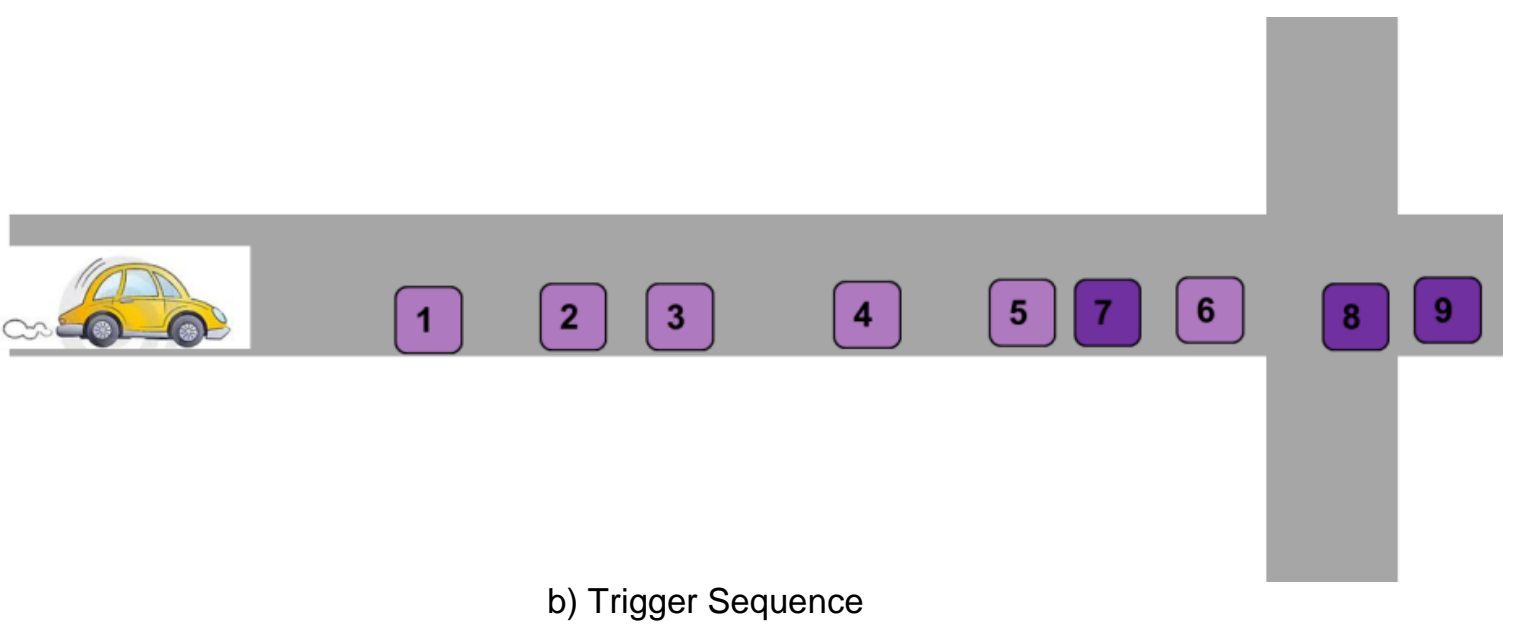

Figure 3. Triggers Layout

\section{Machine Learning Model}

Since statistical learning models do not take into account future resulting states if a certain action was taken, they were not deemed sufficient to model the dynamic aspect of driver's perception of DZ. Reinforcement Learning was considered in this paper as it captures the value of a given state taking into account its Makovian characteristics and transition probabilities [28]. The objective of reinforcement learning algorithms is to find an optimal policy that maps driver's states to their corresponding actions. In our research scope, when an agent is following the optimal policy, it should act close enough to the represented driver's actions. Reinforcement learning are therefore used to train agents to mimic the behavior of a target driver, byt reinforcing agent actions when they perform approximately close to target driver's actions, and penalize actions which are far away from the target actions. The only information available for learning is the system feedback, which describes in terms of rewards and punishment on the task the agent has to realize. In this sense, RL does not only optimize the direct action, but also the total reward the agent can receive in the future.

Reinforcement learning has been applied in driver behavior modeling, network route choice analysis and real time traffic signal control. Neuro-Fuzzy Actor-Critic Reinforcement Learning (NFACRL) approach was used by the authors' research group to combine safety and operation aspects of driver behavior in traffic to model naturalistic driver characteristic and following models [29-37]. Other researchers used feedback reinforcement learning mechanism to model route-choice decision-making under uncertainty [38][39]. In traffic signal optimization research, Abdulhai proposed a Qlearning algorithm in an isolated intersection and then a corridor with coordinated intersections to find the optimal timing plans in a dynamic traffic environment [40, 41].

A major limitation of RL is dealing with continuous data. In this work, we use ActorCritic RL with a fuzzy input layer to map each encountered state to the training set. We use a continuous output (average acceleration) rather than a binary stop or go decision variable. This modeling structure allows us to simulate the driver decision at a microscopic level when needed.

The structure of the actor-critic RL model is shown in Figure 4. Each node in the input (first) layer represents a continuous state variable. We model the driver's decision in DZ as a function of a driver state ahead of the traffic signal. This state is represented in a multi-dimensional space using driver's data as obtained from the driver's simulator. The second layer is the fuzzy membership layer. States are fuzzified in this layer to relate different states to maximum values in the data set. The third layer is the fuzzy rules layer. Each rule is connected with a number of antecedents (discrete fuzzy sets) from the second 
layer. The fourth layer is the discrete action layer including a set of discrete actions for neural network to choose. The output simulated action is the weighted average of the selected actions where fuzzy rule strengths are the associated weights. For a comprehensive description of the method we refer the reader to [34].

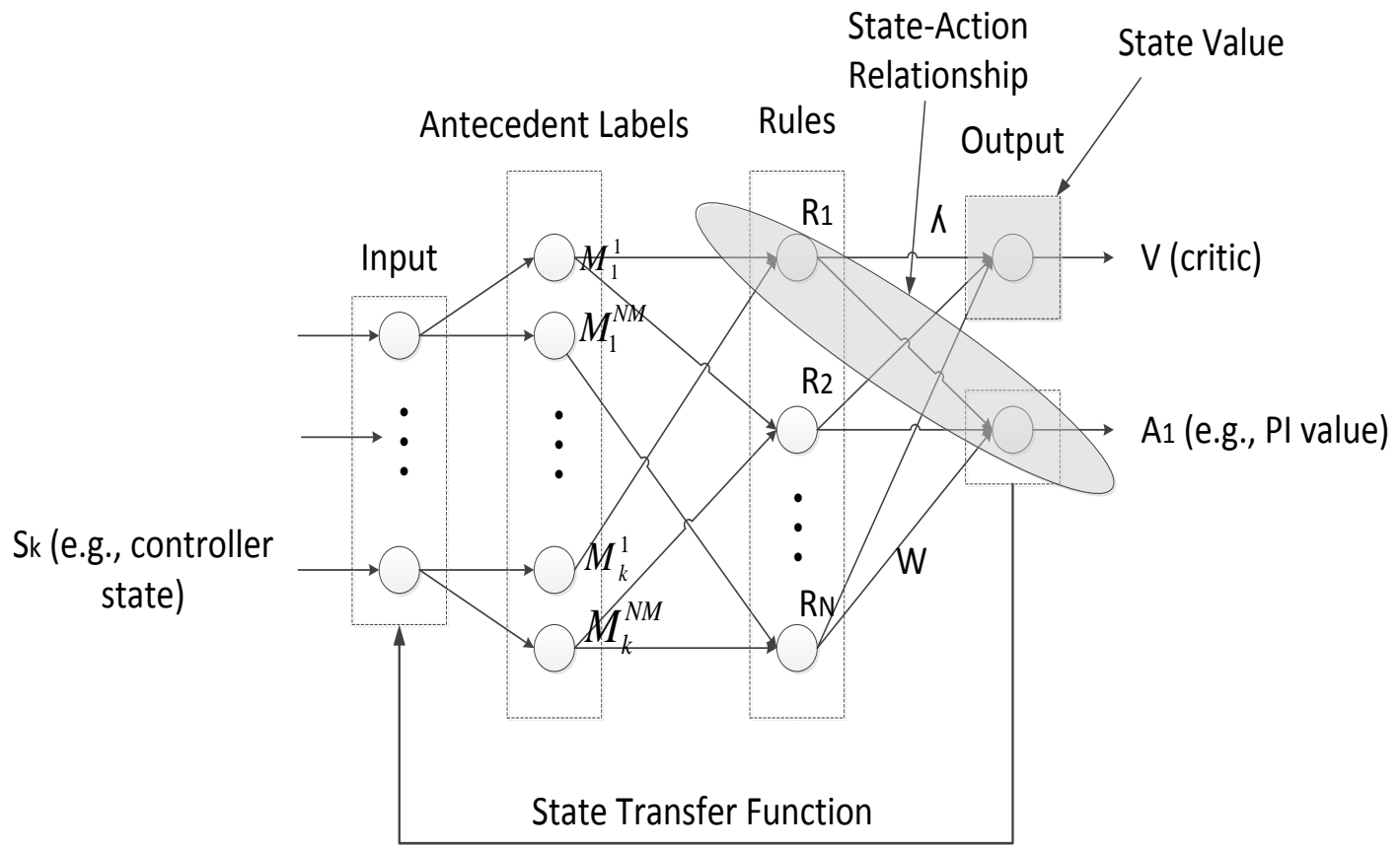

Figure 4. Actor-Critic Neuro-Fuzzy RL Structure

As mentioned earlier, this study included 5 scenarios (each one contains 10 intersections). Data recorded with the precision of $60 \mathrm{~Hz}$ and included speed, longitudinal acceleration, vehicle $\mathrm{x}$ and $\mathrm{y}$ coordinates, time, signal status, and active triggers. A Matlab script was written to manipulate the recorded data and analyze it.

As opposed to many dilemma zone studies that model drivers' decision to stop or go as a binary variable we consider "Mean acceleration" (after yellow indication) as the output variable to construct the model. The reason is that in addition to drivers' decision to stop or go, drivers change in behavior is also important. Alternatively, one would like to know whether the driver stops by hitting the brake hard or choose to stop very smoothly; Or whether the driver pass through the intersection while speeding up or the presence of police make them keep their speed under the speed limit. To be able to address these issues, mean acceleration value is used as an action in this study to capture any behavioral change of driver beyond just stopping and going.

The driver's state is represented in this study by six variables as follows:

$\mathrm{S}_{1}$ : Yellow extension learning variable: this variable keeps track of how many times a driver went through an extended yellow,

$\mathrm{S}_{2}$ : Red-light running variable: this variable keeps track of how many times a driver ran a red light,

$\mathrm{S}_{3}$ : Time to intersection (TTI): time to intersection based on driver's instantaneous speed,

$\mathrm{S}_{4}$ : Time-lost-gained: cumulative time lost or gained starting from six second TTI until the onset of yellow in comparison to constant speed travel time, 
$\mathrm{S}_{5}$ : Mean speed: average speed calculated started from six second TTI until the onset of yellow,

$\mathrm{S}_{6}$ : Mean acceleration: same as above, but for acceleration.

\section{Analysis and Results}

In this study, 400 iterations were run in the training process. Controlling the learning speed of the NFACRL model was handled using three factors, namely memory discount factor $\gamma$, learning factor $\beta$ and reward function scaling factor $\alpha$. The $\gamma$ factor controls the memory fade speed where the value of recently occurring states is weighted more. The $\beta$ factor controls how fast the agent gets the new information. The $\alpha$ factor controls the magnitude of the reward function and weights, and $e_{t h}$ controls the sign of the reward function. In our experiment, we used $\beta=0.6, \gamma=0.9, \alpha=10$, and $e_{t h}=0.2$.

Figure 5 shows the results of the actor-critic agent model. Figure 5-a shows the mean acceleration value for both raw data (circles) and model's output (solid line). Figure 5-b shows a linear fit between model's output and actual data with an $R^{2}$ of 0.72 . The figure shows the output of the model to capture the driver's decision and trace it up and down in a temporal fashion and therefore illustrates the model's capabilities. This was deemed very satisfactory, as it illustrates the ability of the model to capture the driver's experience and to net only outputting mean values for the acceleration as found in the literature.

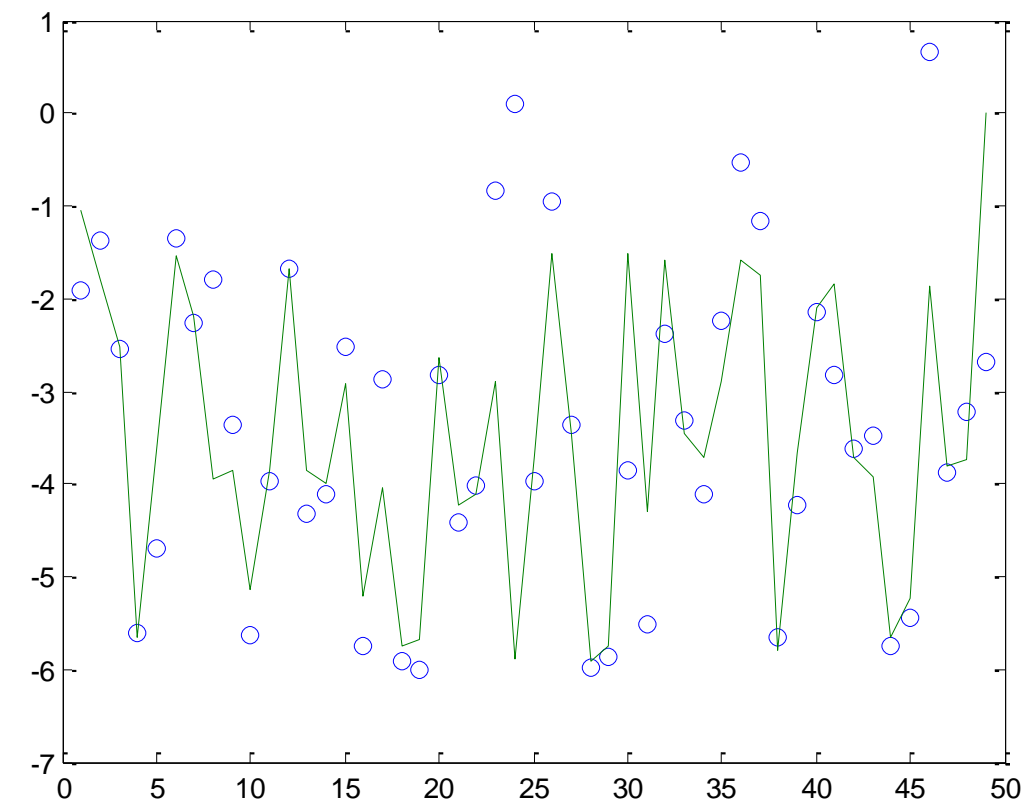

a. Model versus Data for Mean Driver's Acceleration 


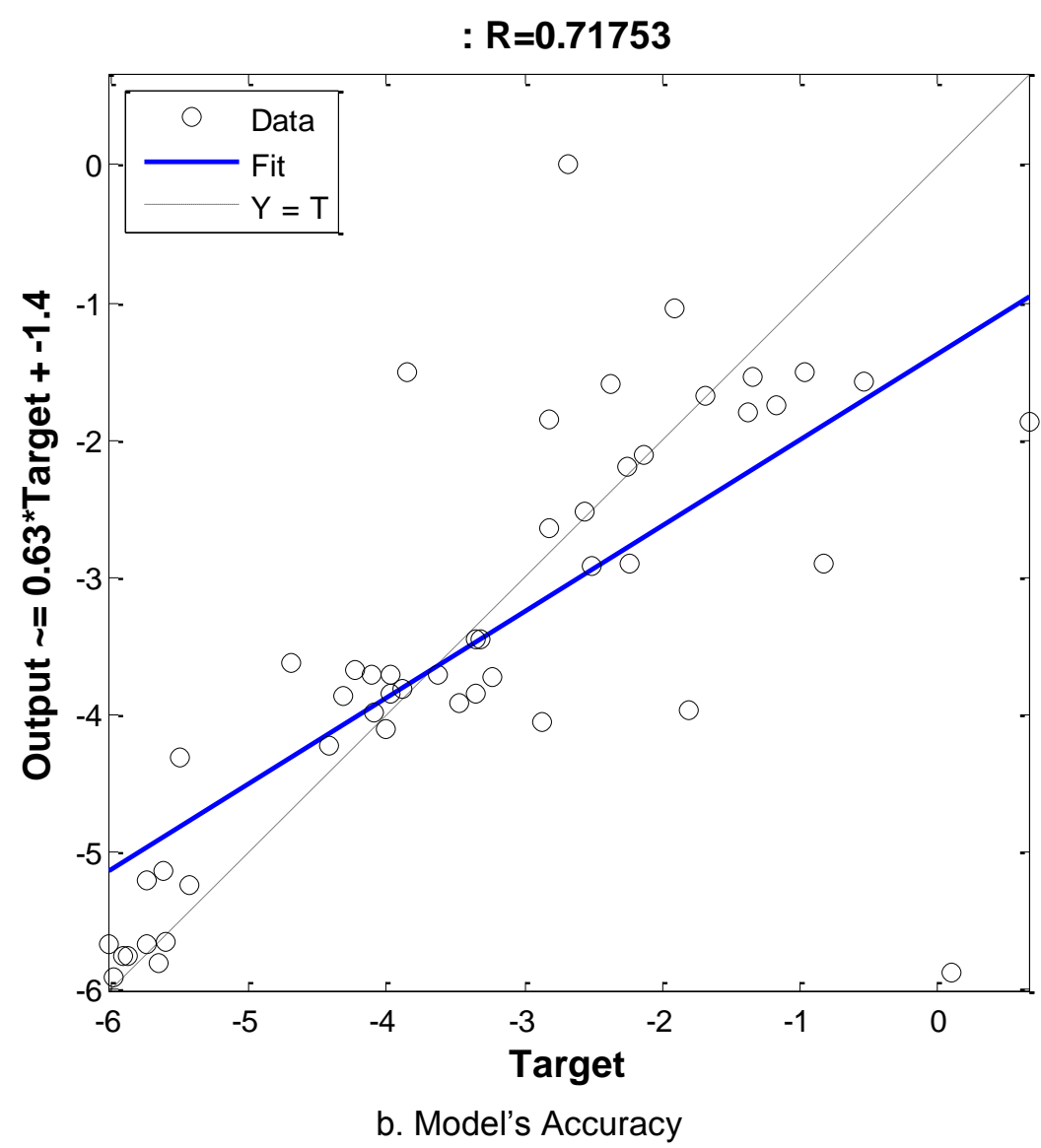

Figure 5. Driver's Actions Results

\section{Conclusions and Future Research}

Several research efforts have addressed dilemma zone mitigation strategies, drivers' perception and reaction in DZ using several modeling techniques. Nevertheless, more research needs to be put into answering how drivers' behavior changes as a result of experience gained from driving through safe and unsafe intersections. To address this research need we implemented an experimental design in a driving simulator environment to explore the dynamic nature of drivers' behavior. In this paper, we implement an actorcritic reinforcement learning algorithm to model the dynamic behavior of driver in dilemma zone. Fuzzy logic was used to partition traffic state variables and a reinforcement learning technique was used for the fuzzy rule policy calibration and update.

This paper contributes to improved modeling of driver behavior in dilemma zone. Consequently, it provides a solid background information to the design of optimal control methods and mitigation strategies for safer intersections. Furthermore, it lays the groundwork for driving simulator studies and scenario development to investigate dilemma zone related issues and drivers' behavior at signalized intersections.

Future research includes the cross-validation analysis of different driver agents. Future work will also include implementation of agent models in a simulated environment for comparative analysis. 


\section{Acknowledgments}

The research undertaken in this paper was sponsored by the Mid-Atlantic Universities Transportation Center. The authors are solely responsible for the material in this paper and the views are not necessarily those of the supporting agency.

\section{References}

[1] K. Zimmerman and J. Bonneson, "In-Service Evaluation of a detection-control system for high-speed signalized intersections". FHWA Report, FHWA/TX-05/5-4022-01-1, (2005).

[2] J. Bonneson, D. Middleton, K. Zimmerman, H. Charara and M. Abbas, "Intelligent Detection-Control System for Rural Signalized Intersections", Texas Transportation Institute Texas A\&M University, (2002).

[3] K. Zimmerman, "Additional Dilemma Zone Protection for Trucks at High-Speed Signalized Intersections", Transportation Research Record: Journal of the Transportation Research Board, 2009(-1): (2007), pp. 82-88.

[4] A. E. Allos and M. I. Al-Hadithi, "Driver behaviour during onset of amber at signalised junctions", Traffic Engineering and Control, vol. 33, no. 5, (1992), pp. 312-317.

[5] C. Liu, R. Herman and D. C. Gazis, "A review of the yellow interval dilemma", Transportation Research Part A: Policy and Practice, vol. 30, no. 5, (1996), pp. 333-348.

[6] P. Papaioannou, "Driver behaviour, dilemma zone and safety effects at urban signalised intersections in Greece", Accident Analysis \&amp; Prevention, vol. 39, no. 1, (2007), pp. 147-158.

[7] N. Elmitiny, X. Yan, E. Radwan, C. Russo and D. Nashar, "Classification analysis of driver's stop/go decision and red-light running violation", Accident Analysis \&amp; Prevention, vol. 42, no. 1, (2010), pp. 101-111.

[8] P. Koonce, L. Rodegerdts, K. Lee, S. Quayle, S. Beaird, C. Braud, J. Bonneson, P. Tarnoff and T. Urbanik, "Traffic signal timing manual", Federal Highway Administration, (2008).

[9] D. S. Hurwitz, "Application of driver behavior and comprehension to dilemma zone definition and evaluation", Open Access Dissertations, (2009), pp. 112.

[10] D. S. Hurwitz, M. A. Knodler and B. Nyquist, "Evaluation of Driver Behavior in Type II Dilemma Zones at High-Speed Signalized Intersections", Journal of transportation engineering, vol. 137, (2011), pp. 277.

[11] D. S. Hurwitz, H. Wang, M. A. Knodler Jr, D. Ni and D. Moore, "Fuzzy sets to describe driver behavior in the dilemma zone of high-speed signalized intersections", Transportation Research Part F: Traffic Psychology and Behaviour, vol. 15, no. 2, (2012), pp. 132-143.

[12] P. L. Olson and R. W. Rothery, "Driver Response to the Amber Phase of Traffic Signals", Operations Research, vol. 9, no. 5, (1961), pp. 650-663.

[13] M.-S. Chang, C. J. Messer and A. J. Santiago, "Timing Traffic Signal Change Intervals Based on Driver Behavior", Transportation Research Board, (1027), (1985), pp. 20-30.

[14] D. Mahalel, D. Zaidel and T. Klein, "Driver's decision process on termination of the green light", Accid Anal Prev, vol. 17, no. 5, (1985), pp. 373-80.

[15] P. L. Olson and R. W. Rothery, "Deceleration levels and clearance times associated with the yellow phase of traffic signals", Traffic Engineering, vol. 42, no. 4, (1972), pp. 16-19, 62-63.

[16] R. H. Wortman and J. S. Matthias, "Evaluation of Driver Behavior at Signalized Intersections", (Discussion), (1983), pp. 0361-1981.

[17] W. L. Williams, "Driver behavior during the yellow interval", Transportation Research Record, (644), (1977), pp. 75-78.

[18] R. Van Der Horst and A. Wilmink, "Drivers' decision-making at signalized intersections: An optimisation of the yellow timing", Traffic Engineering and Control, vol. 27, no. 12, (1986), pp. 615622.

[19] Y. Sheffi and H. Mahmassani, "A model of driver behavior at high speed signalized intersections MA", USA, Transportation science, vol. 15, no. 1, (1981), pp. 50-61.

[20] P. Li and M. Abbas, "Stochastic Dilemma Hazard Model at High-Speed Signalized Intersections", Journal of transportation engineering, vol. 136, no. 5, (2010), pp. 448-456.

[21] T. J. Gates, D. A. Noyce, L. Laracuente and E. V. Nordheim, "Analysis of driver behavior in dilemma zones at signalized intersections", Transportation Research Record: Journal of the Transportation Research Board, 2030, (2007), pp. 29-39.

[22] J. K. Caird, S. L. Chisholm, C. J. Edwards and J. I. Creaser, "The effect of yellow light onset time on older and younger drivers' perception response time (PRT) and intersection behavior", Transportation Research Part F: Traffic Psychology and Behaviour, vol. 10, no. 5, (2007), pp. 383-396.

[23] A. Sharma, D. Bullock and S. Peeta, "Estimating dilemma zone hazard function at high speed isolated intersection", Transportation Research Part C: Emerging Technologies, vol. 19, no. 3, (2011), pp. 400412. 
[24] S. Kikuchi, V. Perincherry, P. Chakroborty and H. Takahashi, "Modeling Of Driver Anxiety During Signal Change Intervals", Transportation Research Record No. 1399, (1993), pp. 27-35.

[25] R. Yager, "Measuring tranquility and anxiety in decision making: An application of fuzzy sets", International Journal of General Systems, vol. 8, (1982), pp. 139-146.

[26] J. Lin and K. Y. Kuo, "Application of fuzzy set theory on the change intervals at a signalized intersection", Applied Soft Computing, vol. 1, no. 2, (2001), pp. 161-177.

[27] S. Ghanipoor Machiani and M. Abbas, "Dynamic Driver's Perception of Dilemma Zone: Experimental Design and Analysis of Driver's Learning in a Simulator Study", Transportation Research Board 93rd Annual Meeting, Wahington DC, (2014).

[28] R. S. Sutton and A. G. Barto, "Reinforcement Learning: An Introduction", 1988, London, England: The MIT Press Cambridge, Massachusetts.

[29] M. Abbas, L. Chong, B. Higgs, A. Medina and C. D. Yang, "Agent-based evaluation of driver heterogeneous behavior during safety-critical events", In Intelligent Transportation Systems (ITSC), 2011 14th International IEEE Conference on IEEE, (2011).

[30] L. Chong, M. Abbas and A. M. Flintsch, "Driver Behavior Simulation Using Agent-based Backpropagation Neural Network", Transportation Research Board 90th Annual Meeting, (2011).

[31] M. M. Abbas, L. Chong, B. Higgs and A. Medina Flintsch, "Agent-Based Reinforcement Learning Model for Simulating Driver Heterogeneous Behavior During Safety-Critical Events in Traffic", Transportation Research Board 91st Annual Meeting, (2012).

[32] L. Chong, M. Abbas, B. Higgs, A. Medina and C. D. Yang, "Determination and optimization of reinforcement learning parameters for driver actions in traffic", Intelligent Transportation Systems (ITSC), 2011 14th International IEEE Conference on IEEE, (2011).

[33] L. Chong, M. Abbas, B. Higgs, A. Medina and C. D. Yang, "A revised reinforcement learning algorithm to model complicated vehicle continuous actions in traffic", Intelligent Transportation Systems (ITSC), 2011 14th International IEEE Conference on IEEE, (2011).

[34] L. Chong, M. M. Abbas, A. Medina Flintsch and B. Higgs, "A rule-based neural network approach to model driver naturalistic behavior in traffic", Transportation Research Part C: Emerging Technologies, (2012).

[35] L. Chong, B. Higgs and A. Medina, "Naturalistic Longitudinal and Lateral Risk-taking Driving Behavior Modeling during Safety-Critical Events".

[36] M. M. Abbas, B. Higgs, L. Chong and A. Medina, "Combined car-following and unsafe event trajectory simulation using agent based modeling techniques", Proceedings of the Winter Simulation Conference, Winter Simulation Conference, (2012).

[37] L. Chong, M. Abbas, B. Higgs, and A. Medina, "Driver Car-following Behavior Simulation using Fuzzy Rule-based Neural Network".

[38] E. Avineri and J. N. Prashker, "Sensitivity to Travel Time Variability: Travelers' Learning Perspective", Transportation Research, Part C:, (2005), pp. 27.

[39] E. A. I. Bogers, M. Bierlaire and S. P. Hoogendoorn, "Modeling Learning in Route Choice", Transportation Research Record, 2014, (2007), pp. 8.

[40] B. Abdulhai, R. Pringle and G. J. Karakoulas, "Reinforcement learning for True Adaptive Traffic Signal Control", Journal of Transportation Engineering, (2003) May-June, pp. 8.

[41] C. Jacob and B. Abdulhai, "Automated adaptive traffic corridor control using reinforcement learning: Approach and case studies", Transportation Research Record, (1959), (2006), p. 1-8.

\section{Authors}

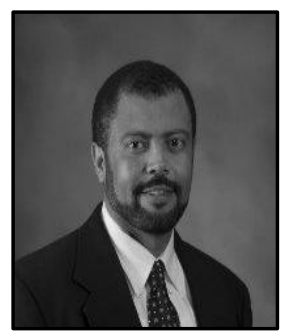

Montasir Abbas is an Associate Professor at Virginia Tech. He received his Ph.D. in Civil Engineering from Purdue University in 2001. He has previously worked as an Assistant Research Engineer at Texas Transportation Institute and as a Visiting Assistant Professor at Texas A\&M. His e-mail is <abbas@vt.edu>.

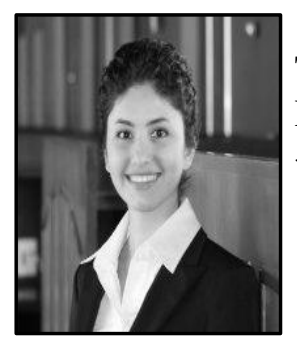

Sahar Ghanipoor Machiani is a Research Associate at Virginia Tech Transportation Institute. She receivedher Ph.D. in Civil Engineering from Virginia Tech in December 2014. Her e-mail is <sahargh@vt.edu>. 
International Journal of Transportation

Vol.4, No.2 (2016) 